\title{
Observation of crystallization slowdown in supercooled parahydrogen and orthodeuterium quantum liquid mixtures
}

\author{
Matthias Kühnel, ${ }^{1}$ José M. Fernández, ${ }^{2}$ Filippo Tramonto, ${ }^{3}$ Guzmán Tejeda, ${ }^{2}$ Elena Moreno, ${ }^{2}$ Anton Kalinin, ${ }^{1}$ \\ Marco Nava, ${ }^{3,4}$ Davide E. Galli, ${ }^{3}$ Salvador Montero, ${ }^{2}$ and Robert E. Grisenti ${ }^{1,5},{ }^{*}$ \\ ${ }^{1}$ Institut für Kernphysik, J. W. Goethe-Universität, Max-von-Laue-Straße 1, 60438 Frankfurt am Main, Germany \\ ${ }^{2}$ Laboratory of Molecular Fluid Dynamics, Instituto de Estructura de la Materia, CSIC, Serrano 121, 28006 Madrid, Spain \\ ${ }^{3}$ Laboratorio di Calcolo Parallelo e di Simulazioni di Materia Condensata, Dipartimento di Fisica, \\ Università degli Studi di Milano, Via Celoria 16, 20133 Milano, Italy \\ ${ }^{4}$ Computational Science, Department of Chemistry and Applied Biosciences, ETH Zurich, USI Campus, \\ Via Giuseppe Buffi 13, CH-6900 Lugano, Switzerland \\ ${ }^{5}$ GSI Helmholtzzentrum für Schwerionenforschung, Planckstraße 1, 64291 Darmstadt, Germany
}

(Received 10 March 2014; published 21 May 2014)

\begin{abstract}
We report a quantitative experimental study of the crystallization kinetics of supercooled quantum liquid mixtures of parahydrogen $\left(\mathrm{pH}_{2}\right)$ and orthodeuterium $\left(\mathrm{oD}_{2}\right)$ by high spatial resolution Raman spectroscopy of liquid microjets. We show that in a wide range of compositions the crystallization rate of the isotopic mixtures is significantly reduced with respect to that of the pure substances. To clarify this behavior we have performed path-integral simulations of the nonequilibrium $\mathrm{pH}_{2}-\mathrm{oD}_{2}$ liquid mixtures, revealing that differences in quantum delocalization between the two isotopic species translate into different effective particle sizes. Our results provide experimental evidence for crystallization slowdown of quantum origin, offering a benchmark for theoretical studies of quantum behavior in supercooled liquids.
\end{abstract}

Understanding the stability of supercooled liquids with respect to crystallization is a fundamental open problem in condensed matter physics [1]. In this regard, since crystallization competes with glass formation, a knowledge of the mechanisms that govern the crystal growth in supercooled liquids is considered an important step to elucidate the nature of the glass transition [2-6]. So far, experimental studies aiming at providing microscopic insights into the dynamics and crystallization of supercooled liquids have been largely based on the use of colloidal suspensions $[7,8]$, where the large particle size allows one to follow the crystal growth on the laboratory time scale. However, diverse drawbacks such as polydispersity and sedimentation often make the experimental data from these systems difficult to interpret $[8,9]$. Accessing the details of the crystallization process in simple atomic and molecular counterparts, on the other hand, remains an experimental challenge due to relevant time scales that are orders of magnitude shorter.

Theoretical studies have shown that the inclusion of quantum effects adds a further degree of complexity in the behavior of supercooled liquids, leading to novel exotic phenomena such as superfluidity $[10,11]$ or enhanced dynamical slowing down [12-14]. Yet again, the difficulties in supercooling a quantum liquid to very low temperatures have so far precluded possible experimental studies of the interplay of quantum effects and structural transformations in nonequilibrium bulk liquids. Here we address these challenges reporting on the experimental investigation of the crystallization kinetics of supercooled liquid mixtures of the isotopic species $\mathrm{pH}_{2}$ and $\mathrm{oD}_{2}$, showing that their quantum nature has a profound impact on the crystallization process.

*grisenti@atom.uni-frankfurt.de
Binary liquid mixtures exhibit, in general, properties that differ fundamentally from their corresponding pure substances, and mixing a few components is, in particular, a common strategy to hinder crystallization. Indeed, classical binary systems of particles that interact via a simple LennardJones (LJ) pair potential have been widely employed as the simplest theoretical models to investigate crystallization and glassy behavior in supercooled liquids [15-19]. Due to the spherical symmetry of the ground-state wave function of the $\mathrm{pH}_{2}$ and $\mathrm{oD}_{2}$ molecules, which are characterized by an even rotational quantum number $J$ [20], a $\mathrm{pH}_{2}-\mathrm{oD}_{2}$ mixture provides a neat molecular binary system in which the pair interactions can be described by the same isotropic $\mathrm{LJ}$ potential [20]. Isotopic $\mathrm{pH}_{2}-\mathrm{oD}_{2}$ mixtures thus combine an intrinsic molecular simplicity with the exciting possibility to explore experimentally quantum behavior in supercooled liquids. It is, in fact, well established that the equilibrium thermodynamic and structural properties of the hydrogen liquids and solids are influenced by quantum effects [21]. The magnitude of quantum effects can be quantified by the dimensionless parameter $\Lambda=\hbar /(\sigma \sqrt{m \epsilon})$, where $\hbar$ is the reduced Planck's constant; $\Lambda$ represents the (effective) de Broglie wavelength of a particle of mass $m$ relative to the length parameter $\sigma$ of the reference $\mathrm{LJ}$ potential characterized by a potential well depth $\epsilon$. For $\mathrm{pH}_{2}$ and $\mathrm{oD}_{2}$ one finds $\Lambda \approx 0.28$ and $\Lambda \approx 0.2$, respectively, which are one order of magnitude larger than the typical values for classical behavior. This quantum character of condensed $\mathrm{pH}_{2}$ and $\mathrm{oD}_{2}$ has led to the prediction of a variety of intriguing effects specific to the hydrogen liquids such as superfluidity of $\mathrm{pH}_{2}$ [10], for which there is so far only indirect evidence coming from spectroscopic studies of small doped $\mathrm{pH}_{2}$ clusters (see, e.g., Ref. [22], and references therein), or the realization of a structural quantum glass in a supercooled $\mathrm{pH}_{2}-\mathrm{oD}_{2}$ mixture [12]. 
To reach a supercooled liquid state we have employed the experimental technique described in Ref. [23]. Briefly, the liquid at equilibrium pressure and temperature is injected into vacuum through a 5- $\mu \mathrm{m}$-diam glass capillary nozzle; the propagating liquid rapidly cools well below the melting temperature until it undergoes a first-order phase transition driven by the onset of homogeneous crystal nucleation, producing a continuous solid filament several centimeters long [23]. A crucial feature of our approach is represented by the univocal correspondence between the distance along the jet propagation direction $z$ and time $t=z / v$, where $v$ is the velocity of the liquid jet. We probed the crystallization kinetics of $\mathrm{pH}_{2}-\mathrm{oD}_{2}$ liquid jets with different $\mathrm{oD}_{2}$ content via Raman light scattering by recording as a function of $z$ (and, thus, as a function of time) spectra of the fundamental vibrational transition, which allows one to distinguish the liquid and solid phases of both the $\mathrm{pH}_{2}$ and $\mathrm{oD}_{2}$ components. The high spatial resolution of the present technique ultimately provides a direct access to the crystallization kinetics on the submicrosecond time scale [23]. The isotopic species $\mathrm{pH}_{2}$ and $\mathrm{oD}_{2}$ were produced by continuous catalytic conversion from $99.9999 \%$ and $99.9 \%$ purity natural $\mathrm{H}_{2}$ and $\mathrm{D}_{2}$, respectively, resulting in $99.8 \%$ and $97.5 \%$ purity $\mathrm{pH}_{2}$ and $\mathrm{oD}_{2}$, respectively, the rest being represented by odd- $J$ molecules. We have investigated pure $\mathrm{pH}_{2}$ and $\mathrm{oD}_{2}$ liquid jets, as well as jets of $\mathrm{pH}_{2}-\mathrm{oD}_{2}$ mixtures with $\mathrm{oD}_{2}$ mole percentages of $1 \pm 0.02$, $3.2 \pm 0.4, \quad 4.6 \pm 0.6, \quad 9.1 \pm 1.2, \quad 16.7 \pm 0.8, \quad 51.6 \pm 1.4$, $83.9 \pm 0.7,95.7 \pm 1.1$, and $97.6 \pm 0.6 \%$. The mixtures were prepared at room temperature by a continuous mixing of the two isotopic gases at the specific ratios set by two mass flow controllers, one for each species, working at a minimum flow rate of $20 \mathrm{nml} \mathrm{min}{ }^{-1}$. The $\mathrm{oD}_{2}$ mole fraction has been further checked by the Raman intensity ratios in the gas (if available) and condensed phases in the vibrational region, confirming the mass flow ratios.

The experimental results are presented in Fig. 1. In Fig. 1(a) we show vibrational Raman spectra for three representative $\mathrm{pH}_{2}-\mathrm{oD}_{2}$ mixtures, clearly evidencing the liquid (L) to solid (S) phase transition. The observed shift to lower wave numbers of the vibrational band corresponding to the liquid with increasing distance from the orifice reflects the evaporative cooling of the propagating filament [23]. This is a consequence of the strong dependence of the vibrational wave number on temperature, though an explicit relation has been determined experimentally only for pure liquid $\mathrm{pH}_{2}$ [24]. In the case of our supercooled $\mathrm{pH}_{2}-\mathrm{oD}_{2}$ mixtures we have found that the vibrational wave number of liquid $\mathrm{pH}_{2}$ at the onset of crystallization shows a linear dependence on the $\mathrm{oD}_{2}$ mole fraction; accordingly, we have estimated the corresponding (average) temperature $T$ (shown by the upper $x$ axis of Fig. 2) by a linear interpolation between the experimental value $T \approx 12 \mathrm{~K}$ for the pure $\mathrm{pH}_{2}$ case [23] and $T \approx 17 \mathrm{~K}$ as computed for pure $\mathrm{oD}_{2}$ by a simple model that reliably describes the evaporative cooling of a liquid jet [23].

In Fig. 1(b) we plot the time evolution of the $\mathrm{pH}_{2}$ and $\mathrm{oD}_{2}$ solid fractions extracted from the respective vibrational Raman spectra with the $\mathrm{oD}_{2}$ mole fraction ranging from 0 (pure $\mathrm{pH}_{2}$ jet, lower curve) to 1 (pure $\mathrm{oD}_{2}$ jet, upper curve). The most striking feature is the remarkable slowdown of the crystallization kinetics with increasing $\mathrm{oD}_{2}$. For example, the presence of only $3 \% \mathrm{oD}_{2}$ molecules leads to nearly twice the time required for the complete freezing of the jet when compared to the pure $\mathrm{pH}_{2}$ case $(\approx 7.9 \mu \mathrm{s})$, as shown in Fig. 2. The slowest crystal growth is observed in the case of the nearly equimolar mixture, which fully crystallizes in $\approx 23.3 \mu$ s, i.e., three times more slowly than the pure $\mathrm{pH}_{2}$ jet. By further increasing the amount of $\mathrm{oD}_{2}$ the duration of the crystallization process then gradually decreases down to $\approx 12.6 \mu$ s for the pure $\mathrm{oD}_{2}$ jet. A second important feature exhibited by the experimental data of Fig. 1(b) is the tendency of the filament to start crystallizing at earlier times with increasing $\mathrm{oD}_{2}$ mole fraction, up to the case of the nearly equimolar mixture. This effect appears at first sight to be in conflict with the subsequent slower crystal growth. However, we can rationalize this behavior in terms of a higher probability for nucleation triggered by purely statistical clustering of $\mathrm{oD}_{2}$ molecules, which reside at a much deeper supercooling than the $\mathrm{pH}_{2}$ molecules with respect to their own melting points. The fact that the beginning of crystallization in mixtures with a higher content of $\mathrm{oD}_{2}$ slightly shifts again towards later times results from the increasingly higher temperature of the filament (see Fig. 2).

The observed dependence of the jet crystallization time on composition as displayed in Fig. 2 is surprising given the isotopic nature of the $\mathrm{pH}_{2}-\mathrm{oD}_{2}$ mixtures. Due to the negligibly small mixing enthalpy [25], which determines the departure of a real mixture from the ideal case, there is no experimental evidence for a phase separation in $\mathrm{H}_{2}-\mathrm{D}_{2}$ mixtures at equilibrium neither in the liquid nor in the solid [25]; this rules out possible effects related to the presence of strongly partitioning species, as observed in supercooled binary metallic alloys [26]. A possible competition between different crystal structures [16,19] seems also unlikely as both $\mathrm{pH}_{2}$ and $\mathrm{oD}_{2}$ crystallize into equilibrium hep crystals [20].

The classical theory of crystal growth [27] is the natural framework to try interpreting our experimental crystallization rates. The crystal growth rate as a function of the temperature is given by $u(T)=k(T)\left\{1-\exp \left[-\Delta G(T) / k_{B} T\right]\right\}$, where $k(T)$ is the crystal deposition rate at the liquid/crystal interface, $\Delta G(T)$ is the difference in Gibbs free energy (per molecule) of the liquid and the crystal, and $k_{B}$ is Boltzmann's constant. For the case of our nearly ideal $\mathrm{pH}_{2}-\mathrm{oD}_{2}$ mixtures $\Delta G(T)$ can be computed starting from the experimental heat capacity data for the pure $\mathrm{pH}_{2}$ and $\mathrm{oD}_{2}$ systems [23]; we find that, for a given temperature, the factor $1-\exp \left[-\Delta G(T) / k_{B} T\right]$ varies only slightly with the amount of $\mathrm{oD}_{2}$ or $\mathrm{pH}_{2}$. Thus, our data suggest that the deposition rate $k(T)$ must be strongly dependent on composition in order to explain the measured crystallization rates. However, identifying this dependence is a challenging task since the coefficient $k(T)$, which reflects collective processes in the liquid, is generally expressed on an empirical basis [2,3,27]. We have recently shown that the crystallization of a pure $\mathrm{pH}_{2}$ filament can be described by the collision-limited model [23], in which the deposition rate scales as $k(T) \propto \sqrt{T / m}$. This mass dependence fairly captures the difference in the crystallization rates observed for the pure $\mathrm{oD}_{2}$ and $\mathrm{pH}_{2}$ jets; the relative duration of the crystallization processes in the two cases would be roughly given by the square root of the deuterium to hydrogen mass ratio, $\approx 1.4$, which is consistent with the experimental value 

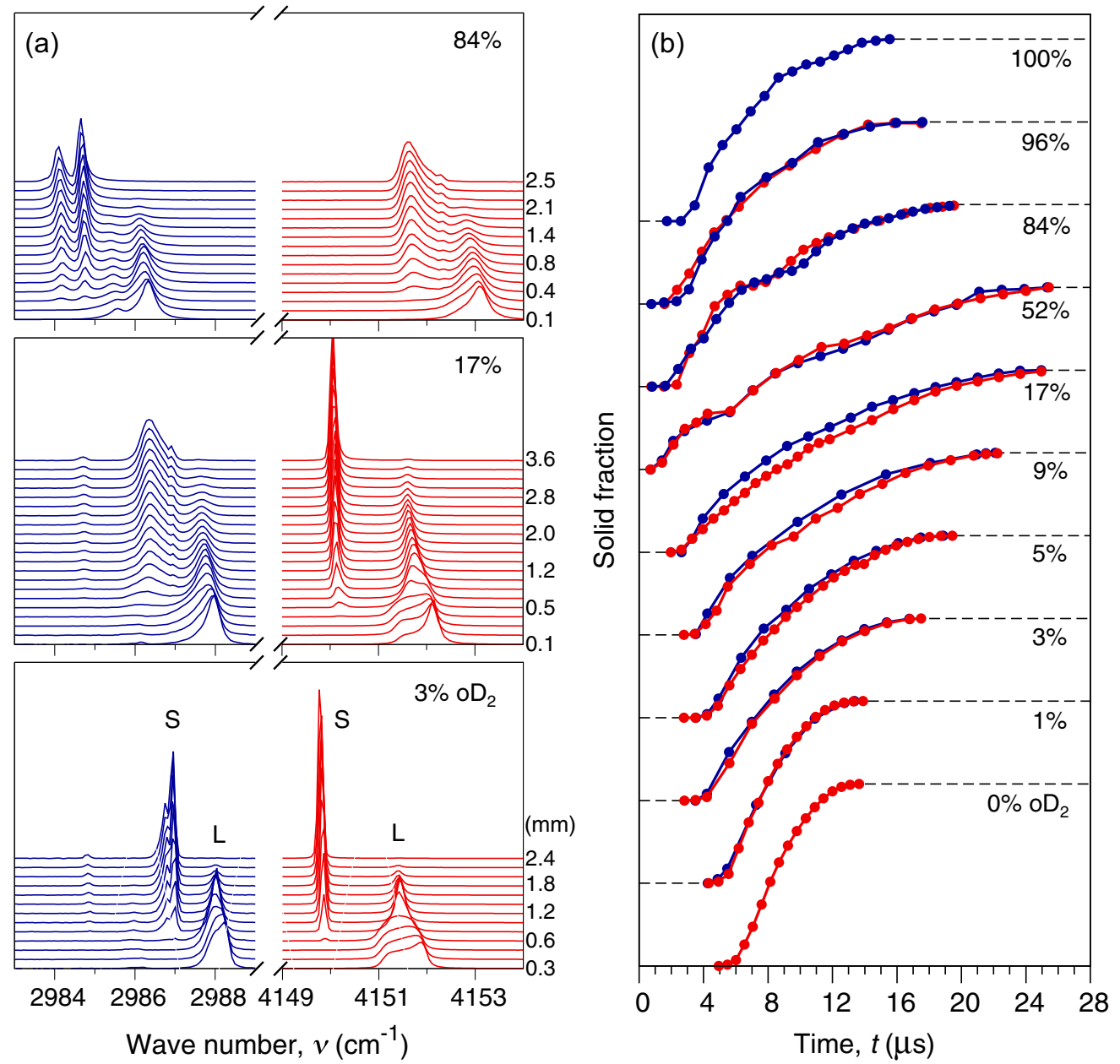

FIG. 1. (Color online) (a) Normalized vibrational Raman spectra of the $\mathrm{oD}_{2}$ (blue, left) and $\mathrm{pH}_{2}$ (red, right) components measured as a function of the distance $z$ from the orifice (right scale) for three representative mixtures. The vibrational bands corresponding to the liquid and solid phases are indicated by L and S, respectively. The double-line shape of the $\mathrm{oD}_{2}$ bands, which is especially evident in the case of the $84 \%$ $\mathrm{oD}_{2}$ mixture, is due to the inevitable presence of less than $3 \%$ of $J=1 \mathrm{pD}_{2}$ molecules, with a 50-fold enhancement in the Raman scattering intensity with respect to the $J=0$ molecules [37]. For the 3 and $17 \% \mathrm{oD}_{2}$ mixtures this enhancement is much smaller and the $J=1$ bands are barely visible in the spectra. (b) Time evolution of the solid fractions extracted from the vibrational bands for $\mathrm{pH}_{2}$ (red points) and oD $\mathrm{D}_{2}$ (blue points), with the time axis defined as $t=z / v$, where $v$ is the jet velocity. The solid fractions range from 0 to 1 , as indicated by the dashed lines on the left and on the right of the experimental curves, respectively.

of $\approx 1.6$ (Fig. 2). However, the above kinetic model is not able to describe the observed dependence of the crystal growth rate on composition, as in this case one would rather expect a monotonic increase of the jet crystallization time with $\mathrm{oD}_{2}$ mole fraction.

As the next step we looked at the bulk structural features, which offer important insights into the behavior of supercooled liquids $[4,15,17,28]$. To access static structural properties of the nonequilibrium $\mathrm{pH}_{2}-\mathrm{OD}_{2}$ quantum liquid mixtures we have carried out path-integral Monte Carlo (PIMC) simulations [29] by using a canonical [30] Worm algorithm [31]. We have simulated mixtures of up to 300 molecules in boxes with periodic boundary conditions with 0,3 , and $10 \% \mathrm{oD}_{2}$ at $T=13 \mathrm{~K}$, with $50 \% \mathrm{oD}_{2}$ at $T=14.5 \mathrm{~K}$, and with 90,97 , and $100 \% \mathrm{oD}_{2}$ at $T=17 \mathrm{~K}$. In order to avoid the crystallization of the mixtures we followed the strategy reported in Ref. [11], verifying that the particles in the simulation cell remained in a disordered metastable configuration for a sufficiently large number $\left(\sim 10^{4}\right)$ of Monte Carlo steps to ensure a reliable characterization of their physical properties. We have also verified that these latter were dependent only on the temperature and the density of the simulated system but not on the particular Monte Carlo stochastic trajectory sampled by checking that the results obtained from statistically independent simulations were consistent with each other.

In Fig. 3(a) we show a snapshot of the classical ring polymers onto which the quantum particles are mapped computed for the $10 \% \mathrm{oD}_{2}$ mixture. The degree of spatial extension of the polymers, each representing a $\mathrm{pH}_{2}$ (red) or $\mathrm{oD}_{2}$ (blue) molecule, is representative of their quantum delocalization; Fig. 3(a) shows that the polymers associated with the $\mathrm{oD}_{2}$ molecules are more compact than those associated with the $\mathrm{pH}_{2}$ molecules. This feature affects the local structural properties, as shown in Fig. 3(b), where we plot the three partial radial pair distribution functions for the average static correlations. We see that the $\mathrm{oD}_{2}-\mathrm{oD}_{2}$ correlation exhibits 


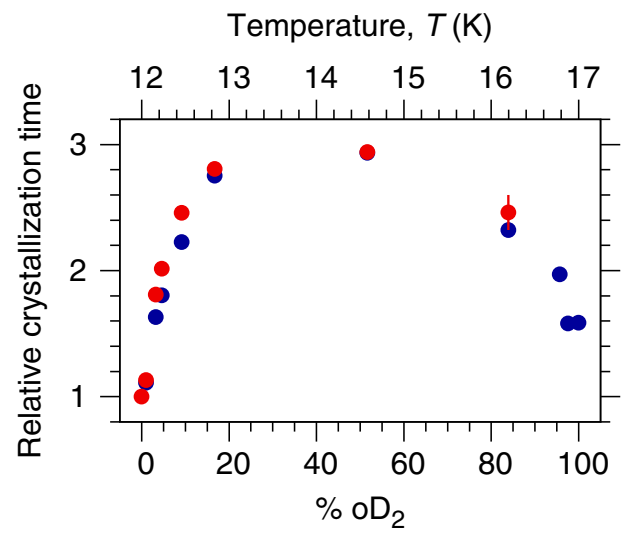

FIG. 2. (Color online) Total duration of the jet crystallization process as determined from the $\mathrm{pH}_{2}$ (red symbols) and $\mathrm{oD}_{2}$ (blue symbols) solid fraction curves of Fig. 1(b). The plotted data indicate relative times with respect to the pure $\mathrm{pH}_{2}$ jet crystallization time of $7.9 \pm 0.4 \mu \mathrm{s}$. The upper $x$ axis represents the estimated average filament temperature at the onset of crystallization as explained in the text.

a higher first peak, which is also shifted towards smaller distances than for the $\mathrm{pH}_{2}-\mathrm{pH}_{2}$ and $\mathrm{pH}_{2}-\mathrm{oD}_{2}$ correlations, indicating significant differences in the average distance between neighboring particles of the two isotopic species. Similar results are found for all the simulated mixtures. We point out that in a classical binary system the assumption of identical pair interactions would lead to identical static correlations for any relative fraction of the two isotopic species [32].

The PIMC simulations show that as a result of the massinduced quantum delocalization the $\mathrm{pH}_{2}$ and $\mathrm{oD}_{2}$ molecules exhibit different "effective sizes," thus illuminating the origin of our measured growth rates. Classical molecular dynamics simulations of binary systems of particles with a given size ratio $[16,19,33]$ have indeed reported a correlation between composition and crystallization kinetics that is strikingly similar to that observed in our experiments. In particular, for binary hard-sphere mixtures it was shown that the crystal growth becomes extremely slow for mole fractions of one of the two components in the range $20 \%-50 \%$ [33]. A similar result was found in the simulation of a model binary LJ system [16], evidencing, in particular, the failure of the mixture to crystallize, i.e., the formation of an amorphous state, for mole fractions of the smaller particles of $20 \%-50 \%$, whereas rapid ordering has been observed otherwise. The similarity between those numerical results and our experimental data, which exhibit a maximum in the jet crystallization time for $\mathrm{oD}_{2}$ mole fractions in the range $20 \%-50 \%$ (Fig. 2), is suggestive, hinting at a common mechanism responsible for the crystallization slowdown. However, a microscopic understanding of how composition and particle size ratio frustrate the crystal growth in binary mixtures is still lacking $[16,19,33]$.

A number of simulation studies $[15,17,18,28]$ have suggested that in one possible scenario crystallization might be hindered by the emergence in the bulk supercooled liquid of locally preferred structures that eventually are incompatible with long-range crystalline order [34]. One important example (a)

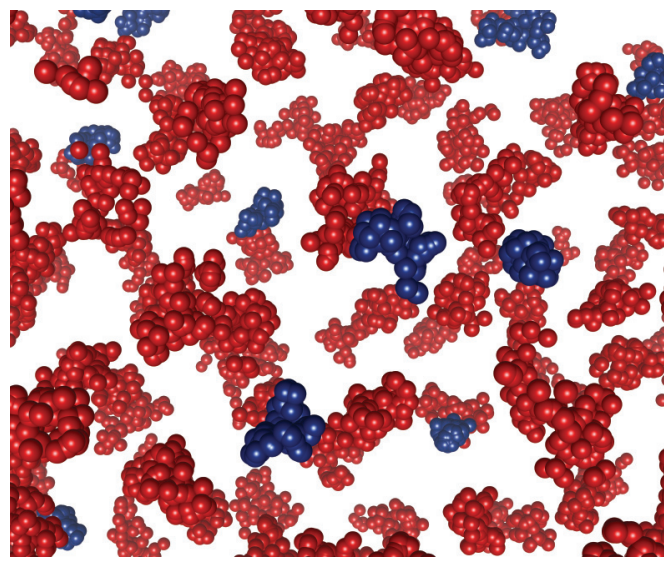

(b)
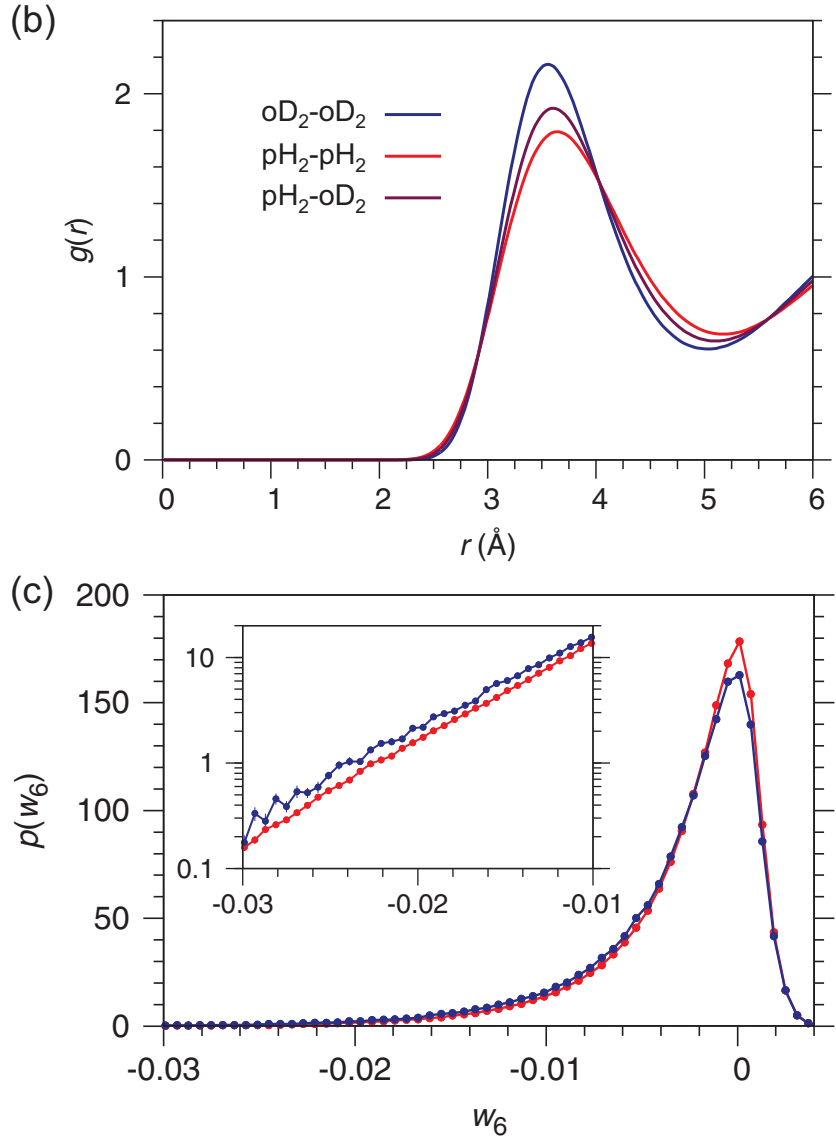

FIG. 3. (Color online) PIMC simulation results for a mixture with $10 \% \mathrm{oD}_{2}$ computed at $T=13 \mathrm{~K}$. (a) Example of a ring-polymer configuration snapshot. The red and blue clusters of particles are the (classical) ring polymers representing the $\mathrm{pH}_{2}$ and $\mathrm{oD}_{2}$ molecules, respectively. (b) Partial radial distribution functions $g(r)$ representing the three pair correlations. (c) Probability distribution $p\left(w_{6}\right)$ for icosahedral-like order for the $\mathrm{pH}_{2}$ (red) and $\mathrm{oD}_{2}$ (blue) molecules. The inset shows an enlarged view of the tail on a logarithmic scale, emphasizing the marked difference between the two isotopic species at high negative values of $w_{6}$.

is the icosahedron with its fivefold symmetry [35], which has been recently found to be a fundamental geometrical motif in the structure of bulk metallic glasses [36]. To explore this 
point we have performed a microscopic structural analysis based on the local bond order invariants method $[4,18,28]$ of our simulated $\mathrm{pH}_{2}-\mathrm{oD}_{2}$ mixtures. We have focused here on the invariant $w_{6}$, which is most sensitive to icosahedral-like order, but the present analysis does not rule out the presence in the metastable liquid of local order with different symmetries. In Fig. 3(c) we plot the probability distribution $p\left(w_{6}\right)$ computed separately for the $\mathrm{pH}_{2}$ and $\mathrm{oD}_{2}$ molecules for the $10 \% \mathrm{oD}_{2}$ mixture. We see a slightly larger tendency for $\mathrm{oD}_{2}$ to populate more negative values of $w_{6}$ than for $\mathrm{pH}_{2}$, denoting an enhanced probability for local noncrystalline order around an $\mathrm{oD}_{2}$ molecule. Similar results are found for all other simulated mixtures. As for the case of classical systems [28], we find that this tendency to noncrystalline order increases with the degree of supercooling. If a correlation between local order and crystal deposition rate could be established, then a difference in the packing efficiency for the two isotopic species as revealed by our structural analysis might provide a physical basis to explain the observed dependence of the crystal growth rate on the $\mathrm{oD}_{2}$ mole fraction; the rearrangement of local noncrystalline structures in the supercooled melt at the liquid/crystal interface would tend to lower the particle diffusivity, thus slowing down the crystal growth [5].
Our experimental results show that composition and particle size ratio play a central role in the kinetics of crystallization of simple nearly-ideal molecular binary mixtures. Understanding the details of this dependence represents the key challenge that should allow one to identify the principles that govern the stability of supercooled liquids against crystallization. With the present work we have not only established a promising experimental route to address this latter fundamental issue, but most notably we have provided evidence for slowdown of crystallization that is purely of quantum origin. In fact, in the supercooled liquid $\mathrm{pH}_{2}-\mathrm{oD}_{2}$ mixtures it is only the difference in the mass-induced quantum delocalization of the two isotopic species that ultimately introduces a degree of frustration of crystallization.

We acknowledge financial support by the Deutsche Forschungsgemeinschaft through Grant No. 593962, and the Spanish Ministerio de Ciencia e Innovación through Grants No. FIS2010-22064-C02 and No. HD2008-0068. We acknowledge CINECA and the Regione Lombardia award, under the LISA initiative, for the availability of high performance computing resources and support. We acknowledge F. Gamez and D. Pini for useful discussions.
[1] M. D. Ediger and P. Harrowell, J. Chem. Phys. 137, 080901 (2012).

[2] M. D. Ediger, P. Harrowell, and L. Yiu, J. Chem. Phys. 128, 034709 (2008).

[3] M. L. F. Nascimento and E. D. Zanotto, J. Chem. Phys. 133, 174701 (2010).

[4] J. Russo and H. Tanaka, Sci. Rep. 2, 505 (2012).

[5] M. Guerdane, H. Teichler, and B. Nestler, Phys. Rev. Lett. 110, 086105 (2013).

[6] C. Tang and P. Harrowell, Nat. Mater. 12, 507 (2013).

[7] U. Gasser, J. Phys.: Condens. Matter 21, 203101 (2009).

[8] G. L. Hunter and E. R. Weeks, Rep. Prog. Phys. 75, 066501 (2012).

[9] H. J. Schöpe, G. Bryant, and W. van Megen, J. Chem. Phys. 127, 084505 (2007).

[10] V. L. Ginzburg and A. A. Sobyanin, JETP Lett. 15, 242 (1972).

[11] O. N. Osychenko, R. Rota, and J. Boronat, Phys. Rev. B 85, 224513 (2012).

[12] E. Rabani and D. R. Reichman, Annu. Rev. Phys. Chem. 56, 157 (2005).

[13] T. E. Markland, J. A. Morrone, B. J. Berne, K. Miyazaki, E. Rabani, and D. R. Reichman, Nat. Phys. 7, 134 (2011).

[14] T. E. Markland, J. A. Morrone, B. J. Berne, K. Miyazaki, E. Rabani, and D. R. Reichman, J. Chem. Phys. 136, 074511 (2012).

[15] D. Coslovich and G. Pastore, J. Chem. Phys. 127, 124504 (2007).

[16] L.-C. Valdes, F. Affouard, M. Descamps, and J. Habasaki, J. Chem. Phys. 130, 154505 (2009).

[17] U. R. Pedersen, T. B. Schrøder, J. C. Dyre, and P. Harrowell, Phys. Rev. Lett. 104, 105701 (2010).
[18] S. Jungblut and C. Dellago, J. Chem. Phys. 134, 104501 (2011).

[19] A. Banerjee, S. Chakrabarty, and S. M. Bhattacharyya, J. Chem. Phys. 139, 104501 (2013).

[20] I. F. Silvera, Rev. Mod. Phys. 52, 393 (1980).

[21] M. Zoppi, J. Phys.: Condens. Matter 15, R1047 (2003).

[22] T. Zeng and P.-N. Roy, Rep. Prog. Phys. 77, 046601 (2014).

[23] M. Kühnel, J. M. Fernández, G. Tejeda, A. Kalinin, S. Montero, and R. E. Grisenti, Phys. Rev. Lett. 106, 245301 (2011).

[24] R. Sliter and A. F. Vilesov, J. Chem. Phys. 131, 074502 (2009).

[25] D. White and J. R. Gaines, J. Chem. Phys. 42, 4152 (1965).

[26] D. M. Herlach and P. K. Galenko, Mater. Sci. Eng. A 449, 34 (2007).

[27] K. A. Jackson, Inter. Sci. 10, 159 (2002).

[28] M. Leocmach, J. Russo, and H. Tanaka, J. Chem. Phys. 138, 12A536 (2013).

[29] D. M. Ceperley, Rev. Mod. Phys. 67, 279 (1995).

[30] M. Rossi, M. Nava, L. Reatto, and D. E. Galli, J. Chem. Phys. 131, 154108 (2009).

[31] M. Boninsegni, N. V. Prokof'ev, and B. V. Svistunov, Phys. Rev. E 74, 036701 (2006).

[32] A. P. Lima, A. S. Martins, and J. S. Sá Martins, Physica A 391, 4281 (2012).

[33] S. R. Williams, C. P. Royall, and G. Bryant, Phys. Rev. Lett. 100, 225502 (2008).

[34] G. Tarjus, S. A. Kivelson, Z. Nussinov, and P. Viot, J. Phys.: Condens. Matter 17, R1143 (2006).

[35] F. C. Frank, Proc. R. Soc. London, Ser. A 215, 43 (1952).

[36] A. Hirata, L. J. Kang, T. Fujita, B. Klumov, K. Matsue, M. Kotani, A. R. Yavari, and M. W. Chen, Science 341, 376 (2013).

[37] B. J. Kozioziemski and G. W. Collins, Phys. Rev. B 67, 174101 (2003). 